\title{
resultados de las mediciones de polvo en hormos de cemento
}

\author{
G. FUNKE Y H. A. FISCHER \\ Instituto de Investigación de la industria del Cemento, Düsseldorf \\ Zement-Kalk-Gips, n॰ 4, abril 1967
}

\section{INTRODUCCION}

Desde 1950, el Servicio de Información de la industria cementera realiza mediciones de polvo en fábricas de cemento y otras industrias similares. De acuerdo con las exigencias de la pureza del aire, que en los últimos años han crecido considerablemente, aumenta cada vez más el número de mediciones de polvo. Así, en el año 1966, se examinó la emisión de polvo en 37 fábricas de cemento; se estudiaron 79 instalaciones y se realizaron 270 medidas.

Gran parte de las mediciones se realizaron por encargo de las autoridades estatales relacionadas con la inspección de industrias, ya que, en varios Estados confederados de Alemania, el Servicio de Información del Instituto está reconocido oficialmente para efectuar estas medidas. Otras mediciones sirvieron para estudiar la garantía dada por las casas vendedoras sobre los aparatos de desempolvamiento, después de la puesta en servicio de las nuevas instalaciones. Finalmente, también se midió la emisión de polvo por encargo de las fábricas, así como la eficacia de las instalaciones de desempolvado después de un largo tiempo de funcionamiento o después de hechas algunas transformaciones en la instalación.

A continuación se expondrán los resultados de mediciones de polvo en hornos de cemento equipados con instalaciones de recuperación de polvo, de acuerdo con los últimos adelantos de la técnica.

Los valores que figuran en los cuadros se refieren, siempre que no se indique explícitamente otra cosa, a las condiciones gaseosas en el punto de medida, es decir, detrás del recuperador de polvo y, en muchos casos, en la chimenea de la instalación.

Las mediciones se realizaron con las instalaciones en funcionamiento continuo y sin previo examen general de los filtros.

\section{RESULTADO DE LAS MEDICIONES}

\subsection{Hornos rotatorios de vía húmeda con electrofiltro}

Los resultados de las mediciones de polvo en hornos rotatorios largos de vía húmeda fi- 
guran en las tablas 1a y 1b. El electrofiltro del horno núm. 1 está equipado con una cámara de chapa de acero, con dos campos de tensión y dos grupos de alta tensión; todavía no está terminada una tercera zona en la entrada del filtro. En este horno, las condiciones del gas para el electrofiltro eran muy ventajosas. Los hornos núms. 2 y 3 están equipados cada uno con un electrofiltro de 2 cámaras de hormigón en paralelo. También, en estos casos, cada una de las cámaras está dotada de dos campos de tensión y una zona vacía suplementaria. El electrofiltro del horno núm. 2 tiene un grupo especial para cada uno de los campos de tensión, o sea, cuatro grupos en total. Las mediciones de polvo realizadas en este filtro demostraron que con sólo dos grupos, sin regulación de tensión automática, no se podía lograr un contenido de polvo en el gas depurado completamente satisfactorio. El horno núm. 3 tiene dos grupos separados, para limpieza previa y posterior de las cámaras. El contenido de polvo del gas depurado es también más elevado que en el horno núm. 2.

T A B L A 1a

Hornos rotatorios largos de vía húmeda con filtro eléctrico

\begin{tabular}{|c|c|c|c|c|c|c|c|}
\hline Núm. & $\begin{array}{c}\text { Cantidad de gases } \\
\text { de escape } \\
\left(\mathrm{Nm}^{3} / \mathrm{h}\right)\end{array}$ & $\begin{array}{c}\text { Cantidad de gases } \\
\text { de escape } \\
\text { especifica } \\
\left(\mathrm{Nm}^{3} / \mathbf{k g} \text { Kli }\right)\end{array}$ & $\begin{array}{l}\text { Tempe- } \\
\text { ratura } \\
(o c)\end{array}$ & $\begin{array}{l}\text { Punto } \\
\text { de rocio } \\
\text { (oc) }\end{array}$ & $\begin{array}{l}\mathrm{CO}_{2} \\
(\%)\end{array}$ & $\begin{array}{c}\mathbf{O}_{2} \\
(\%)\end{array}$ & $\begin{array}{c}\text { Contenido } \\
\text { de polvo en los } \\
\text { gases depurados } \\
\left(\mathrm{g} / \mathrm{Nm}^{3}\right)\end{array}$ \\
\hline 1 & 94.000 & 3,48 & 133 & 70 & 22,4 & 6,2 & 0,103 \\
\hline $2 a$ & 148.700 & 3,95 & 153 & 74 & 22,9 & 4,3 & 0,145 \\
\hline $2 b$ & 139.200 & 3,69 & 151 & 74 & 22,1 & 5,0 & 0,090 \\
\hline $3 a$ & 158.800 & 4,15 & 156 & 68 & 17,6 & 8,4 & 0,226 \\
\hline $3 b$ & 144.300 & 3,77 & 160 & 68 & 18,8 & 6,2 & 0,176 \\
\hline
\end{tabular}

T A B L A $1 b$

\begin{tabular}{|c|c|c|c|c|c|c|}
\hline \multirow[t]{2}{*}{ Núm. } & \multirow{2}{*}{$\begin{array}{c}\text { Temperatura } \\
\text { cámara } \\
\text { de humos } \\
(\circ)\end{array}$} & \multirow{2}{*}{$\begin{array}{l}\text { Temperatura } \\
\text { delante ciel } \\
\text { electrofiltro } \\
\text { (oC) }\end{array}$} & \multirow{2}{*}{$\begin{array}{c}\text { Contenido } \\
\text { de polvo } \\
\text { del gas sucio } \\
\left(\mathrm{g} / \mathrm{Nm}^{3}\right)\end{array}$} & \multirow{2}{*}{$\begin{array}{c}\text { Grado de } \\
\text { separación } \\
(\%)\end{array}$} & \multicolumn{2}{|c|}{ Grupos del electrofiltro } \\
\hline & & & & & $\begin{array}{c}\text { Tensión } \\
\text { (V) }\end{array}$ & $\begin{array}{l}\text { Intensidad } \\
\quad(\mathbf{m A})\end{array}$ \\
\hline 1 & 155 & 137 & 3,75 & 97,25 & $266 / 210$ & $189 / 190$ \\
\hline \multirow[t]{2}{*}{$2 \mathrm{a}$} & \multirow[t]{2}{*}{162} & \multirow[t]{2}{*}{158} & \multirow[t]{2}{*}{3,79} & \multirow[t]{2}{*}{96,2} & $306 / 302$ & $156 / 163$ \\
\hline & & & & & $333 / 388$ & $362 / 353$ \\
\hline \multirow[t]{2}{*}{$2 b$} & \multirow[t]{2}{*}{160} & \multirow[t]{2}{*}{156} & \multirow[t]{2}{*}{3,79} & \multirow[t]{2}{*}{96,2} & $305 / 302$ & $155 / 159$ \\
\hline & & & & & $331 / 337$ & $362 / 356$ \\
\hline $3 a$ & 177 & 174 & 6,54 & 96,6 & $407 / 375$ & $227 / 258$ \\
\hline $3 b$ & 176 & 173 & 9,30 & 98,1 & $413 / 378$ & $248 / 273$ \\
\hline
\end{tabular}

Las diferencias en el contenido de polvo del gas depurado entre los núms. $2 \mathrm{a}$ y $2 \mathrm{~b}$ se deben a que en el $2 \mathrm{~b}$ la cantidad de gases de escape era más baja ya que, simultáneamente, en una limpieza posterior, el golpeo de los electrodos de precipitación se cambió de permanente a intermitente, porque era de temer que el polvo muy ligero fuera arrastrado en cada golpe por la corriente de gas. 
Eń el horno núm. 3 se observa claramente que una cantidad de gases de escape más elevada por introducción de aire falso (3a) conduce a una elevación del contenido de polvo en el gas depurado.

En los últimos años ya no se construyen hornos rotatorios de vía húmeda con secador de pasta (calcinador, concentrador), pero los que aún se encuentran en servicio se han equipado con filtros eléctricos, puesto que el grado de desempolvamiento de los aparatos centrífugos ya no respondían a las exigencias actuales. Los resultados de las mediciones de polvo están resumidos en la tabla 2.

T A B L A 2

Horno rotatorio de vía húmeda con secador de pasta (concentrador, calcinador) $y$ electrofiltro

\begin{tabular}{|c|c|c|c|c|c|c|c|c|c|}
\hline \multirow[t]{2}{*}{ Núm. } & \multirow{2}{*}{$\begin{array}{l}\text { Cantidad } \\
\text { de gases } \\
\text { de escape } \\
\left(\mathrm{Nm}^{3} / \mathrm{h}\right)\end{array}$} & \multirow{2}{*}{$\begin{array}{c}\text { Cantidad } \\
\text { de gases } \\
\text { de escape } \\
\text { especifica } \\
\left(\mathbf{N m}^{3} / \mathbf{k g} \mathbf{K l i}\right)\end{array}$} & \multirow{2}{*}{$\begin{array}{l}\text { Tempe- } \\
\text { ratura } \\
\left({ }^{\circ} \text { C) }\right.\end{array}$} & \multirow{2}{*}{$\begin{array}{c}\text { Punto } \\
\text { de rocío } \\
(o C)\end{array}$} & \multirow{2}{*}{$\begin{array}{l}\mathrm{CO}_{2} \\
(\%)\end{array}$} & \multirow{2}{*}{$\begin{array}{l}\mathbf{O}_{2} \\
(\%)\end{array}$} & \multirow{2}{*}{$\begin{array}{l}\text { Contenido } \\
\text { de polvo } \\
\text { en el gas } \\
\text { depurado } \\
\left(\mathrm{g} / \mathbf{N m}^{3}\right)\end{array}$} & \multicolumn{2}{|c|}{ Grupos del electrofiltro } \\
\hline & & & & & & & & (V) & $(\mathrm{mA})$ \\
\hline 1 & 62.300 & 6,00 & 194 & 66 & 15,4 & 8,7 & 0,140 & $410 / 135$ & $215 / 227$ \\
\hline 2 & 59.100 & 3,94 & 132 & 66 & 23,0 & 7,3 & 0,090 & $66 / 57 \mathrm{kV}$ & $140 / 242$ \\
\hline 3 & 89.900 & 5,37 & 121 & 64 & 12,0 & 11,4 & 0,132 & $498 / 460$ & $118 / 275$ \\
\hline 4 & 124.000 & 4,81 & 127 & 72 & 20,5 & 6,0 & 0,047 & $500 / 490$ & $270 / 530$ \\
\hline
\end{tabular}

Los electrofiltros de una cámara están equipados cada uno con dos campos de tensión y dos grupos de alta tensión.

El electrofiltro del horno núm. 1, una instalación antigua con una gran proporción de aire parásito en los gases de escape y una alta temperatura en los mismos, sólo podía mantener un valor por debajo de $0,15 \mathrm{~g} / \mathrm{Nm}^{3}$, no reintroduciendo el polvo recuperado en la alimentación del horno, con lo que el contenido de polvo del gas sucio se reducía en más de la mitad.

En el horno núm. 2 y tres hornos más de la misma factoría, los electrofiltros están dimensionados de tal forma que, incluso con una gran cantidad de polvo en el gas sucio, tal como ocurre cuando se retorna polvo en la entrada de horno, puedan separar cantidades de polvo elevadas en el gas contaminado. Con ensayos de breve duración encaminados a reducir el contenido de polvo del gas sin depurar por evacuación del polvo separado, se logró reducir la circulación de polvo en más de la mitad, pero apenas se consiguió influir el contenido de polvo en el gas depurado. Cuando al variar la circulación de polvo se perturbaba la marcha del horno, el efecto del electrofiltro era incluso pernicioso. También los hornos núms. 3 y 4 , siendo instalaciones antiguas, tienen una gran cantidad específica de gases de escape. Se observa que la escasa proporción de aire falso en los gases de escape del horno núm. 4, con punto de rocío y contenido de $\mathrm{CO}_{2}$ más elevados, repercute favorablemente en el electrofiltro.

Mediciones recientes en hornos rotatorios de vía húmeda antiguos con inyección de pasta han mostrado que, sólo mediante la modernización y ampliación de los electrofiltros, se 
pueden conseguir contenidos de polvo en el gas depurado por debajo de $0,15 \mathrm{~g} / \mathrm{Nm}^{3}$. Pero puesto que en estos hornos las condiciones de polvo y gases de escape pueden variar rápidamente, sucesivas mediciones de polvo han dado con frecuencia resultados diferentes.

\subsection{Hornos Lepol con electrofiltro}

Los hornos Lepol pueden desempolvarse bien mediante electrofiltros. puesto que las condiciones de los gases de escape y del polvo son favorables, aunque los filtros deban protegerse suficientemente contra corrosiones.

En la tabla 3 se ha hecho una selección de gran número de resultados de las mediciones realizadas. Se ha dado preferencia a instalaciones con condiciones particularmente interesantes. Los electrofiltros se componen, por regla general, de una o, en caso de grandes cantidades de gas, de dos cámaras de hormigón, con dos campos de tensión cada una. Mientras que los filtros antiguos poseen a menudo sólo un grupo de alta tensión, en las instalaciones más modernas se prevén frecuentemente dos grupos para conseguir un efecto de desempolvamiento más elevado.

T A B L A 3

Hornos Lepol con electrofiltro

\begin{tabular}{|c|c|c|c|c|c|c|c|c|c|}
\hline \multirow[t]{2}{*}{ Núm. } & \multirow{2}{*}{$\begin{array}{l}\text { Cantidad } \\
\text { de gases } \\
\text { de escape } \\
\left(\mathrm{Nm}^{3} / \mathrm{h}\right)\end{array}$} & \multirow{2}{*}{$\begin{array}{c}\text { Cantidad } \\
\text { de gases } \\
\text { de escape } \\
\text { específica } \\
\left(\mathrm{Nm}^{3} / \mathrm{kg} \mathrm{Kli}\right)\end{array}$} & \multirow{2}{*}{$\begin{array}{l}\text { Tempe- } \\
\text { ratura } \\
(\text { oc) }\end{array}$} & \multirow{2}{*}{$\begin{array}{c}\begin{array}{c}\text { Punto } \\
\text { de rocio }\end{array} \\
\left({ }^{\prime}(\mathrm{C})\right.\end{array}$} & \multirow{2}{*}{$\begin{array}{l}\mathrm{CO}_{2} \\
(\%)\end{array}$} & \multirow{2}{*}{$\begin{array}{l}\mathbf{o}_{2} \\
(\%)\end{array}$} & \multirow{2}{*}{$\begin{array}{l}\text { Contenido } \\
\text { de polvo } \\
\text { en el gas } \\
\text { depurado } \\
\left(\mathrm{g} / \mathrm{Nm}^{3}\right)\end{array}$} & \multicolumn{2}{|c|}{ Grupos del electrofiltro } \\
\hline & & & & & & & & (V) & $(\mathbf{m A})$ \\
\hline 1 & 48.900 & 2,17 & 122 & 62 & 22,9 & 5,6 & 0,053 & $58 / 58 \mathrm{kV}$ & $33 / 95$ \\
\hline $2 a$ & 69.300 & 2,36 & 88 & 55 & 22,0 & 8,5 & 0,054 & $255 / 209$ & $243 / 243$ \\
\hline $2 b$ & 65.500 & 2,21 & 81 & 59 & 23,3 & 7,3 & 0,124 & $225 / 215$ & $218 / 190$ \\
\hline $3 a$ & 52.400 & $3,00 *)$ & 97 & 49 & 15,3 & 11,3 & 0,150 & 336 & 253 \\
\hline $3 b$ & 55.300 & $2,88^{* \prime}$ & 99 & 59 & 18,0 & 9,0 & 0,086 & 370 & 221 \\
\hline $4 a$ & 69.700 & 2,32 & 98 & 58 & 19,7 & 8,4 & 0,060 & $4.65 / 435$ & $88 / 169$ \\
\hline $4 b$ & 72.200 & 2,41 & 98 & 58 & 19,7 & 8,4 & 0,119 & $-/ 417$ & $-/ 75$ \\
\hline 5 & 106.000 & $\left.2,95^{*}\right)$ & 92 & 54 & 17,8 & 10,2 & 0,142 & $286 / 378$ & $206 / 230$ \\
\hline 6 & 104.200 & 2,57 & 118 & 57 & 19,0 & 8,7 & 0,096 & $472 / 482$ & $142 / 117$ \\
\hline 7 & 142.000 & 2,56 & 97 & 59 & 20,1 & 8,1 & 0,031 & $450 / 448$ & $372 / 378$ \\
\hline $8 a$ & 198.500 & 2,39 & 101 & 57 & 20,0 & 6,8 & 0,033 & 420 & $20 \mathrm{~A}$ \\
\hline $8 b$ & 192.000 & 2,31 & 97 & 57 & 17.6 & 9,4 & 0,140 & 400 & $15 \mathrm{~A}$ \\
\hline
\end{tabular}

(*) Cantidad de gases de escape adicional del plato granulador y de los transportadores de crudo.

El horno núm. 1 tiene, para sus dimensiones, una producción de clínker muy elevada. Las condiciones de servicio son constantes, puesto que, a pesar de la elevada temperatura en la parrilla Lepol, los gránulos plásticos no revientan. Con una propórción de aire falso relativamente escasa en los gases de escape del horno y un punto de rocío elevado, se midió un contenido de polvo en el gas depurado muy reducido.

En el horno núm. 2, se realizaron medidas de garantía (2a), unos 6 meses después de la 
puesta en servicio del electrofiltro. Después de 3 años de servicio, se repitieron estas mediciones (2b), comprobándose que el contenido de polvo en los gases depurados, después del electrofiltro, había subido a más del doble, a pesar de que las condiciones de los gases de escape para el electrofiltro habían mejorado algo; esto obligó a realizar un examen exhaustivo y una revisión completa del electrofiltro.

También en el horno núm. 3 se repitieron las mediciones de polvo después de 3 años, encontrándose el electrofiltro siempre en buen estado. En la medida de repetición (3b) se obtuvo un contenido de polvo $\epsilon \mathrm{n}$ el gas depurado considerablemente más bajo, ya que el punto de rocío y el contenido de $\mathrm{CO}_{2}$ de los gases eran más favorables para el electrofiltro, por el cambio de la combustión con carbón a fuel-oil y por la eliminación de aire falso con una producción más elevada del horno. La elevada cantidad de gases de escape específica se debe a que se mezclan unos $7.000 \mathrm{Nm}^{3} / \mathrm{h}$ de aire procedentes del escape del filtro de mangas del plato granulador y transportador de crudo con los gases de escape del horno, delante del filtro eléctrico.

El electrofiltro del horno núm. 4 está equipado con dos grupos (4a). Cuando al efectuar una medición (4b), sólo estaba en servicio la limpieza posterior, el contenido de polvo del gas depurado subió al doble. A pesar de ello, se mantuvo la garantía de filtración, puesto que el electrofiltro estaba dimensionado para esta eventualidad (avería en un grupo). Al conmutar un grupo a ambos campos de tensión, casi se alcanzaron los valores a pleno servicio con dos grupos.

En el horno núm. 5 se mezclan $25.700 \mathrm{Nm}^{3} / \mathrm{h}$ de aire de escape del plato granulador, transportador de crudo y de la pesada de éste (con un contenido de polvo de $9,8 \mathrm{~g} / \mathrm{Nm}^{3}$ ), con los gases de escape del horno, delante del electrofiltro. De esta forma, las condiciones de servicio de dicho electrofiltro se perturbaron considerablemente y el contenido de polvo del gas d purado no resultó satisfactorio. Por esto se recomendó a la Empresa desempolvar este aire de escape en un filtro de tejido y no introducirlo a continuación en el electrofiltro.

En la instalación núm. 6, las averías en la regulación de tensión automática de los grupos del electrofiltro de doble cámara fueron la causa de que en las mediciones no se alcanzase el contenido de polvo en el gas depurado de acuerdo con la garantía. Después de la reparación efectuada en un grupo, se consiguieron valores de tensión e intensidad mucho mejores en una cámara del filtro, de forma que el contenido de polvo descendió por debajo de $0,08 \mathrm{~g} / \mathrm{Nm}^{3}$.

El electrofiltro de la instalación núm. 7 está construido como filtro de hormigón vertical con dos campos de tensión en paralelo y dos grupos. El contenido de polvo del gas depurado en marcha normal de la instalación era muy favorable.

La instalación núm. 8 está constituida por dos hornos Lepol desempolvados por un electrofiltro común. Mientras que en servicio normal (8a) el contenido de polvo en el gas depurado era muy escaso, aumentó fuertemente durante una medición (8b), porque en la parrilla Lepol se sustituyeron algunas placas que provocaron la entrada de aire falso y un servicio irregular del filtro con frecuentes saltos de chispas y valores de tensión e intensidad bajos.

\subsection{Horno rotatorio con precalentador de gas en suspensión y electrofiltro}

El desempolvamiento con electrofiltro de los hornos rotatorios con precalentadores de gas 
de suspénsión se considera particularmente difícil, a causa de emplear sólo una parte de los gases de escape para el secado y producirse una temperatura de mezcla desfavorable de $180^{\circ}$ a $250^{\circ} \mathrm{C}$ en el filtro o, también, por emplearlo en "servicio directo" sin aprovechamiento de gases cuando las condiciones son desfavorables.

En muchas instalaciones, en servicio combinado con el secadero, se consigue sin dificultad un contenido de polvo del gas depurado inferior a $100 \mathrm{mg} / \mathrm{Nm}^{3}$; pero en servicio directo, la expulsión de polvo resulta de cinco a diez veces superior si los gases no se humectan por inyección de agua en el precalentador o en una torre de refrigeración separada y se enfrían por debajo de la temperatura crítica.

En la tabla 4 se exponen algunos resultados de medición particularmente interesantes realizados en estas instalaciones.

T A B L A 4

Hornos rotatorios con precalentadores de gas en suspensión y electrofiltros

\begin{tabular}{|c|c|c|c|c|c|c|c|c|c|c|}
\hline \multirow[t]{2}{*}{ Núm. } & \multirow{2}{*}{$\begin{array}{c}\text { Cant1dad } \\
\text { de gases } \\
\text { de escape } \\
\left(\mathrm{Nm}^{3} / \mathrm{h}\right)\end{array}$} & \multirow{2}{*}{$\begin{array}{c}\text { Cantidad } \\
\text { de gases } \\
\text { de escape } \\
\text { especifica } \\
\left(\mathrm{Nm}^{3} / \mathbf{k g ~ K l i}\right)\end{array}$} & \multicolumn{2}{|c|}{ 'Temperatura } & \multirow{2}{*}{$\left|\begin{array}{c}\text { Punto } \\
\text { de } \\
\text { rocío } \\
(o c)\end{array}\right|$} & \multirow{2}{*}{$\begin{array}{l}\mathbf{C O}_{2} \\
(\%)\end{array}$} & \multirow{2}{*}{$\begin{array}{l}\mathbf{O}_{2} \\
(\%)\end{array}$} & \multirow{2}{*}{$\begin{array}{c}\text { Contenido } \\
\text { de polvo } \\
\text { en el gas } \\
\text { depurado } \\
\left(\mathrm{g} / \mathrm{Nm}^{3}\right)\end{array}$} & \multicolumn{2}{|c|}{$\begin{array}{l}\text { Grupos del } \\
\text { electrofiltro }\end{array}$} \\
\hline & & & $\begin{array}{c}\text { Chimenea } \\
\text { (oc) }\end{array}$ & 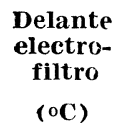 & & & & & (v) & $(\mathrm{mA})$ \\
\hline $\left.1^{*}\right)$ & 26.000 & 1,47 & 273 & 313 & 40 & 29,9 & 3,7 & 0,129 & $300 / 310$ & $70 / 210$ \\
\hline $\left.2 a^{* *}\right)$ & 87.800 & 3,19 & 108 & 165 & 57 & 15,2 & 11,8 & 0,072 & $327 / 347$ & $300 / 285$ \\
\hline $\left.2 b^{* * *}\right)$ & 56.000 & 2,03 & 174 & 237 & 55 & 22,6 & 7,0 & 0,350 & $300 / 300$ & $300 / 300$ \\
\hline $\left.3 a^{* *}\right)$ & 59.000 & 1,89 & 131 & 157 & 48 & 23,8 & 6,7 & 0,033 & $272 / 264$ & $490 / 492$ \\
\hline $\left.3 b^{* * *}\right)$ & 45000 & 1,46 & 250 & 320 & 52 & 31,5 & 2,4 & 0,230 & $209 / 181$ & $490 / 510$ \\
\hline $4 * * *)$ & 56.900 & 1,78 & 189 & 195 & 58 & 31,2 & 2,8 & 0,216 & $300 / 295$ & $574 / 510$ \\
\hline $\left.5 a^{* *}\right)$ & 180.000 & 2,71 & 91 & 100 & 56 & 23,2 & 7,5 & 0,044 & $\begin{array}{l}495 / 495 \\
495 / 495\end{array}$ & $\begin{array}{l}290 / 300 \\
312 / 333\end{array}$ \\
\hline $\left.5 b^{* * *}\right)$ & 173.750 & 2,61 & 127 & 140 & 52 & 20,6 & 8,5 & 0,233 & $\begin{array}{l}388 / 412 \\
427 / 420\end{array}$ & $\begin{array}{r}62 / 130 \\
155 / 160\end{array}$ \\
\hline $\left.6^{* * *}\right)$ & 178.200 & 2,37 & 91 & 100 & 56 & 21,6 & 8,8 & 0,059 & $477 / 488$ & $82 / 107 \mathrm{~A}$ \\
\hline $\left.7 a^{* *}\right)$ & 242.000 & 2,69 & 96 & 110 & 38 & 14,9 & 11,8 & 0,084 & $312 / 335$ & $170 / 770$ \\
\hline $\left.7 b^{* * * *}\right)$ & 179.000 & 1,99 & 144 & 165 & 45 & 22,2 & 7,8 & 0,067 & $280 / 320$ & $140 / 750$ \\
\hline
\end{tabular}

(*) Servicio directo sin inyección de agua.

(**) Con aprovechamiento de gases de escape en instalaciones de secado o de molienda con secado simultáneo.

$(* * *)$ Servicio directo con inyección de agua.

La expulsión de polvo del horno núm. 1 se midió durante una prueba de horno. Las condiciones para el electrofiltro eran particularmente favorables. Las mediciones sólo se realizaron en servicio directo, ya que no había secadero acoplado. Por tanto, la proporción de aire falso en los gases de escape era muy escasa y la temperatura superior a $320^{\circ} \mathrm{C}$. La resistencia eléctrica del polvo era, como resultó de las medidas de la firma suministradora, más favorable en este caso que lo es en general con polvos de estos hornos en otras factorías. 
En la instalación núm. 2 se aprovechan los gases de escape en servicio normal en una instalación de molienda con secado (2a). Pero según el contenido de humedad de las materias primas, hay que conducir una determinada cantidad de gas directamente al electrofiltro. Mediante la inyección de 1 a $2 \mathrm{~m}^{3} / \mathrm{h}$ de agua en el intercambiador de calor, se consigue que la temperatura delante del electrofiltro sea inferior a unos $170^{\circ} \mathrm{C}$ y que el contenido de polvo del gas depurado sea bajo. Los domingos y días festivos se trabaja sin aprovechamiento de los gases de escape (2b); entonces se inyectan de 3,5 a $4 \mathrm{~m}^{3} / \mathrm{h}$ de agua con lo cual se logra delante del electrofiltro una temperatura de los gases de $230^{\circ}$ a $240^{\circ} \mathrm{C}$. Una mayor cantidad de agua en el intercambiador de calor reduciría la temperatura de los gases por debajo de $200^{\circ} \mathrm{C}$; así se mejora el contenido de polvo del gas limpio, pero después de unas horas de servicio se producen dificultades en el funcionamiento del horno. Puesto que la proporción de aire falso en los gases de escape es muy elevada, cabe la posibilidad de algunas mejoras.

El electrofiltro del horno núm. 3 está equipado con un enfriador por evaporación de escasa altura y se halla bajo una ligera sobrepresión. La cantidad de aire falso es, por tanto, escasa. En servicio con aprovechamiento de los gases de escape (3a), resulta un contenido de polvo en el gas depurado muy bajo. En servicio directo (3b), el efecto de la torre de refrigeración es pequeño, puesto que hasta ahora no se consiguió inyectar una cantidad de agua superior a $1,2 \mathrm{~m}^{3} / \mathrm{h}$, sin que se produjeran depósitos y hasta lodo en la torre de refrigeración. Con el montaje de nuevas toberas regulables y una mejor distribución del gas en la torre de refrigeración se intenta mejorar el acondicionamiento de los gases.

En la instalación núm. 4 se realizó la medición en servicio directo, conduciéndose la tubería de los gases de escape a una instalación de molienda con secado simultáneo. Los ventiladores-exhaustores de los gases de escape del horno y de desempolvado después del electrofiltro se manejaban de tal forma que se aspiraba la mínima cantidad posible de aire falso. En el intercambiador se inyectó la mayor cantidad de agua posible $\left(4,8 \mathrm{~m}^{3} / \mathrm{h}\right)$, de forma que la temperatura de los gases delante del filtro fuera inferior a $200^{\circ} \mathrm{C}$ y el punto de rocío resultase elevado.

Los gases de escape del horno núm. 5 se conducen desde el intercambiador, a través de un tambor de secado. El electrofiltro conectado a con`inuación tiene dos cámaras en paralelo con dos campos de tensión cada una y en total cuatro grupos de alta tensión. Trabajando el secadero (5a), las condiciones de trabajo del electrofiltro son muy favorables.

Sin introducción de material en el secadero, o sea, en servicio directo (5b), se inyectan unos $8 \mathrm{~m}^{3}$ de agua por hora en el tambor; así se consigue un grado de separación satisfactorio en el filtro. La proporción de aire falso es todavía bastante elevada como consecuencia de los numerosos puntos no estancos del tambor de secado.

En el horno núm. 6, unos dos tercios de la cantidad de gases de escape pasan a través de dos instalaciones de molienda con secado simultáneo; el resto pasa a un enfriador por evaporación elevado. Los gases se reúnen delante del electrofiltro en una cámara de mezclado. La torre de refrigeración está dimensionada de tal modo que, en caso de perturbaciones en el servicio de molienda, pueda absorber la cantidad total de gases del horno, enfriándola por debajo de $170^{\circ} \mathrm{C}$ y humectándola de forma que, incluso en estas condiciones, se obtiene un buen rendimiento del electrofiltro.

La instalación núm. 7 está equipada de forma análoga a la anterior, con la diferencia que en el servicio normal de ésta se aprovecha la casi totalidad de los gases de escape en las instalaciones de secado y molienda con secado simultáneo (7a). En este caso, la proporción de aire parásito en los gases de escape después del secado es muy elevada. En 
servicio directo se inyectan en un enfriador por evaporación de grandes dimensiones hasta unos $15 \mathrm{~m}^{3}$ de agua por hora, de modo que se establece una temperatura delante del filtro inferior a $170^{\circ} \mathrm{C}$, sin depósitos o formación de lodo en la torre de refrigeración. $\mathrm{El}$ rendimiento del filtro es muy bueno, porque simultáneamente la cantidad de gases de escape y la proporción de aire falso son más reducidas y el punto de rocío y contenido de $\mathrm{CO}_{2}$ de los gases más elevado. Condiciones para el servicio sin perturbaciones del enfriador por evaporación son, aparte de un buen aislamiento y una buena distribución del gas, un funcionamiento seguro y regulable de las toberas y una buena conservación de la instalación.

Las mediciones de polvo realizadas en hornos rotatorios con precalentadores de gas de suspensión y equipados con electrofiltros dimensionados para el servicio normal con aprovechamiento de los gases de escape, han demostrado que sólo en raras ocasiones se han medido contenidos bajos de polvo en el gas depurado cuando se emplean en servicio directo sin aprovechamiento de calor. Con una inyección de agua escasa, pero sin perturbaciones en el intercambiador de calor o en el conducto de gases de escape, la temperatura apenas desciende por debajo del valor crítico para la resistencia del polvo. Sólo con una temperatura de entrada en el filtro por debajo de $170^{\circ}-180^{\circ} \mathrm{C}$ mediante una inyección de mayor proporción de agua, se puede esperar un rendimiento satisfactorio; en servicio continuo no se podrá inyectar en el sistema de gases de escape la cantidad de agua necesaria, no se utiliza un gran enfriador por evaporación.

Al no disponer de agua suficiente para acondicionar los gases de escape y al utilizar el electrofiltro durante largo tiempo en servicio directo, hay que dimensionar la instalación para este caso de explotación. Mediante una construcción amplia de las cámaras con escasa velocidad del gas y tiempo de permanencia del mismo muy largo y división del filtro en varios campos de tensión con grupos separados, se deben de intentar vencer las condiciones desfavorables para gases y polvo.

Para el servicio del filtro es importante que la proporción de aire falso en la corriente de los gases de escape sea lo más reducida posible. Un gran contenido de $\mathrm{CO}_{2}$ y un elevado punto de rocío con escasa cantidad de gases repercuten favorablemente en el electrofiltro.

\subsection{Hornos verticales con electrofiltro}

En los últimos años se han desarrollado también electrofiltros para hornos verticales, aunque todavía hay pocas instalaciones en servicio en la República Federal Alemana. Los valores de medición expuestos en la tabla 5 muestran una buena idea del efecto de estos filtros.

T A B L A 5

Hornos verticales con electrofiltro

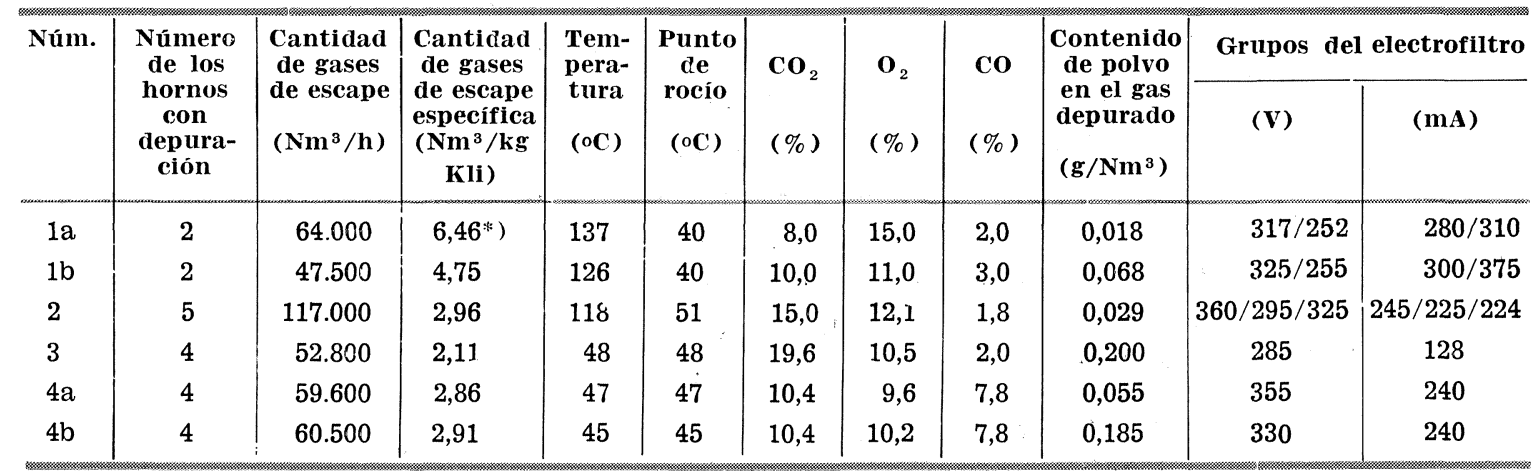

(*) Con el aire expulsado del enfriador de un horno Lepol. 
La instalación núm. 1 se compone de dos hornos verticales, cuyos gases de escape se mezclan en una cámara con el aire procedente del enfriador de un horno Lepol y se calientan para ser desempolvados a continuación en un electrofiltro (1a). El polvo se separa muy bien, como lo demuestra el escaso contenido del mismo en el gas depurado.

Al parar el horno Lepol, los gases de escape del horno vertical pueden calentarse mediante un sistema de calefacción con fuel-oil en la cámara de mezcla (1b). En este estado de explotación, la fuerte fluctuación de la temperatura de los gases de escape del horno vertical (entre $50^{\circ}$ y $150^{\circ} \mathrm{C}$ ) repercutió desfavorablemente en el servicio del electrofiltro, puesto que la regulación de la calefacción con fuel-oil era demasiado lenta para poder establecer con suficiente rapidez el equilibrio en la temperatura.

Los gases de escape de los cinco hornos verticales de la instalación núm. 2 se mantuvieron, mediante una proporción más elevada de combustible en los nódulos, a una temperatura media en la cámara de mezcla delante del electrofiltro de unos $120^{\circ} \mathrm{C}$. El electrofiltro dimensionado correctamente, compuesto de dos cámaras en paralelo con tres campos de tensión cada una y tres grupos de alta tensión en total, eliminó el polvo de los gases de escape de los hornos tan perfectamente que ya no se veía el penacho de humo color blanco azulado, típico de los hornos verticales.

Dentro de un proyecto de investigación, también se realizaron mediciones en electrofiltros humectados para hornos verticales.

El electrofiltro irrigado de la instalación núm. 3 desempolva cuatro hornos verticales, cuya temperatura de gases de escape se sitúa alrededor del punto de rocío. Si bien el contenido de polvo del gas depurado no era satisfactorio, puesto que el filtro es demasiado pequeño, se pudo distinguir el efecto de filtrado por el penacho de humo considerablemente reducido de la chimenea.

También en la instalación núm. 4 se desempolvan cuatro hornos verticales con un electrofiltro humectado bastante mayor que el filtro de la instalación núm. 3. Hubo dificultades para mantener limpias las toberas de aspersión en el electrofiltro. Mientras que con las toberas limpias y a plena circulación de agua con unos $52 \mathrm{~m}^{3} / \mathrm{h}$ se consiguió un contenido de polvo en el gas depurado muy bajo (4a), al atascarse unas toberas (4b) descendieron tanto la cantidad de agua como el rendimiento del electrofiltro, lo cual se manifestó por una presión superior en la tubería.

Pronto se dispondrá de más resultados de medidas de polvo realizadas en electrofiltros con calentamiento de los gases de escape, con fuel-oil, ya que en la actualidad se están construyendo o poniendo en servicio varias instalaciones.

\subsection{Aire que escapa de los enfriadores de parrilla}

También debe desempolvarse el aire de escape de los enfriadores de los hornos rotatorios, sobre todo cuando se evacua a través de una chimenea directamente al aire libre. Como recuperadores de polvo se emplean multiciclones y, en casos especiales, filtros de lecho granular. El rendimiento de estos aparatos depende sobre todo de la granulometría del polvo, de la carga de filtro y de la conservación de las instalaciones.

En la tabla 6 se resumen los resultados de las medidas de polvo realizadas en instalaciones modernas. 
T A в L A 6

Aire de escape de enfriadores de parrilla

\begin{tabular}{|c|c|c|c|c|c|c|c|}
\hline Núm. & $\begin{array}{c}\text { Cantidad } \\
\text { de aire } \\
\text { de escape } \\
\left(\mathrm{Nm}^{3} / \mathrm{h}\right)\end{array}$ & $\begin{array}{c}\text { Cantidad } \\
\text { de aire } \\
\text { de escape } \\
\text { especifica } \\
\left(\mathrm{Nm}^{3} / \mathrm{kg} \mathrm{Kli}\right)\end{array}$ & $\begin{array}{c}\text { Tempera- } \\
\text { tura } \\
\left({ }^{\circ} \mathrm{C}\right)\end{array}$ & $\begin{array}{l}\text { Contenido } \\
\text { de polvo } \\
\text { en el gas } \\
\text { depurado } \\
\left(\mathrm{g} / \mathrm{Nm}^{3}\right)\end{array}$ & $\begin{array}{l}\text { Contenido } \\
\text { de polvo } \\
\text { en el gas } \\
\text { sucio } \\
\left(\mathrm{g} / \mathrm{Nm}^{3}\right)\end{array}$ & $\begin{array}{c}\begin{array}{c}\text { Grado } \\
\text { de } \\
\text { depura- } \\
\text { ción }\end{array} \\
(\%)\end{array}$ & $\begin{array}{c}\text { Clase } \\
\text { de } \\
\text { aparato } \\
\text { depurador }\end{array}$ \\
\hline $1 \mathrm{a}$ & 31.000 & 1,58 & 249 & 0,033 & 3,10 & 98,9 & Multiklon \\
\hline $1 b$ & 32.900 & 1,70 & 269 & 0,071 & - & - & - \\
\hline 2 & 36.900 & 1,08 & 256 & 0,201 & 6,27 & 96,8 & Multiklon \\
\hline 3 & 42.600 & 1,28 & 247 & 0,164 & 9,63 & 98,3 & Multiklon \\
\hline 4 & 56.500 & 1,63 & 222 & 0,072 & 9,98 & 99,3 & Multiklon \\
\hline 5 & 56.600 & 1,81 & 227 & 0,078 & - & - & Multiklon \\
\hline 6 & 95.850 & 1,24 & 223 & 0,220 & - & - & Multiklon \\
\hline $7 a$ & 47.700 & 1,73 & 161 & 0,023 & 2,33 & 99,1 & $\begin{array}{l}\text { Filtro } \\
\text { de lecho }\end{array}$ \\
\hline $7 b$ & 61.700 & 2,24 & 191 & 0,217 & - & - & granular \\
\hline
\end{tabular}

La primera medición (1a) en la instalación núm. 1 tuvo lugar poco tiempo después de la puesta en servicio: En una medición (1b) realizada después de un año, el contenido de polvo a la salida del multiciclón había subido, pero quedaba muy por debajo de $100 \mathrm{mg} / \mathrm{Nm}^{3}$.

En la instalación núm. 2, el polvo tiene una propensión extraordinaria a depositarse en los multiciclones. El contenido de polvo indicado en la tabla 6 se había determinado poco después de una limpieza de los ciclones. Cuando dichos ciclones no se vigilan durante cierto tiempo, el contenido de polvo de esta instalación sube considerablemente.

El recuperador de multiciclones de la instalación núm. 3 se compone de dos grupos de ciclones que durante las medidas tenían una admisión irregular, probablemente debido a la conformación del canal de aire sucio que une el enfriador con el recuperador de polvo. Además había puntos en cortocircuito entre el aire sucio y el aire depurado en una mitad del depurador.

En la instalación núm. 4, el contenido de polvo del aire de escape de enfriador se midió en la chimenea común a dos hornos. Las dos instalaciones de multiciclones se limpiaron en intervalos periódicos, de modo que en servicio normal de la instalación realizan una buena depuración.

Las compuertas de descarga (válvula de doble charnela) de los multiciclones en la instalación núm. 5 se renovaron antes de iniciarse las mediciones de polvo, pues las compuertas existentes no cerraban bien, habiendo bajado el rendimiento del depurador por esta causa.

Después de las mediciones de polvo en la instalación núm. 6 se comprobó, durante una parada del horno, que una parte de los multiciclones estaba atascada con trozos de clínker y depósitos, lo cual explicaba el contenido de polvo residual, no del todo satisfactorio.

El aire de escape del enfriador de la instalación núm. 7 se depuró en un filtro de lecho granular con 36 cámaras. Con una cantidad de aire de escape normal del horno y una carga superficial de filtro neta de unos $1.500 \mathrm{~m}^{3}$ por $\mathrm{m}^{2}$ de superficie de filtro y hora, el contenido de polvo del gas filtrado es escaso (7a). 
En otro ensayo (7b) se aumentó la cantidad de aire de escape en un $30 \%$ aproximadamente, de forma que resultó una carga en el filtro de casi $2.200 \mathrm{~m}^{3} / \mathrm{m}^{2}$. h. El contenido de polvo en el gas depurado subió bastante, lo que demuestra hasta qué punto el grado de separación del filtro de lecho granular depende de la cantidad de gases; a su vez, según el tipo y cantidad del polvo, no se puede sobrepasar una determinada carga superficial del filtro.

\section{RESUMEN}

Se presentan, bajo forma de tablas, los resultados de las medidas en diverso tipo de instalaciones para dar una idea de la situación actual de lás técnicas de depuración de polvo en los hornos de cemento.

Una buena disposición y concepción de los dispositivos de depuración permite, la mayor parte de las veces, respetar los valores límites exigidos en el momento actual para el contenido en polvo de los gases depurados.

No obstante, el estado de la técnica no impide que, a menudo, surjan ciertas desviaciones que alteran el grado de depuración logrado.

El acondicionamiento de los gases de combustión mediante agua pulverizada es siempre difícil, en particular en el caso de hornos rotatorios con precalentadores en suspensión gaseosa cuando trabajan directamente acoplados al electrofiltro.

Evitar las entradas de aire falso y una adecuada y continuada conservación, contribuyen mucho a asegurar que los colectores de polvo mantengan la eficiencia requerida en condiciones de trabajo continuadas. 